\title{
OPEN Optimized preoperative determination of nerve of origin in patients with vestibular schwannoma
}

\begin{abstract}
Torsten Rahne ${ }^{1,3 \boxplus}$, Stefan K. Plontke ${ }^{1}$, Laura Fröhlich ${ }^{1}$ \& Christian Strauss $^{2}$
In vestibular schwannoma (VS) patients hearing outcome and surgery related risks can vary and depend on the nerve of origin. Preoperative origin differentiation between inferior or superior vestibular nerve may influence the decision on treatment, and the selection of optimal treatment and counselling modalities. A novel scoring system based on functional tests was designed to predict the nerve of origin for VS and was applied to a large number of consecutive patients. A prospective, double blind, cohort study including 93 patients with suspected unilateral VS was conducted at a tertiary referral centre. Preoperatively before tumor resection a functional test battery [video head-impulse test (VHIT) of all semicircular canals (SCC)], air-conducted cervical/ocular vestibular evoked myogenic potential tests (cVEMP, oVEMP), pure-tone audiometry, and speech discrimination was applied. Sensitivity and specificity of prediction of intraoperative finding by a preoperative score based on VHIT gain, cVEMP and oVEMP amplitudes and asymmetry ratios was calculated. For the prediction of inferior vestibular nerve origin, sensitivity was $73 \%$ and specificity was $80 \%$. For the prediction of superior vestibular nerve origin, sensitivity was $60 \%$ and specificity was $90 \%$. Based on the tradeoff between sensitivity and specificity, optimized cut-off values of - 0.32 for CVEMP and -0.11 for oVEMP asymmetry ratios and vHIT gain thresholds of 0.77 (anterior SCC), 0.84 (lateral SCC) and 0.80 (posterior $\mathrm{SCC}$ ) were identified by receiver operator characteristic curves. The scoring system based on preoperative functional tests improves prediction of nerve of origin and can be applied in clinical routine.
\end{abstract}

Cochleovestibular schwannomas, usually termed as 'vestibular schwannomas' or 'acoustic neuromas', are benign tumors that most often develop in the human internal auditory canal and the cerebellopontine angle, and sometimes in the labyrinth ${ }^{1}$. Formerly thought to originate from Schwann cells in the glial-Schwannian transitional zone of the vestibulocochlear nerve, cochleovestibular schwannomas do in fact arise anywhere along the eighth cranial nerve $\mathrm{e}^{2-4}$. The majority of cochleovestibular schwannomas present in the internal auditory canal (IAC) with or without extension to the cerebello-pontine angle (CPA). In these locations, they most often arise from the superior (SVN) or inferior vestibular nerve (IVN) $)^{5-7}$, while for the rarer intralabyrinthine schwannomas the cochlea is the site of origin in at least $50 \%$ of the cases ${ }^{8}$. This article focuses on the "classical" vestibular schwannoma (VS) in the IAC/CPA.

Patients with VS often experience early auditory symptoms, such as hearing loss and tinnitus and vestibular dysfunctions can occur with increasing tumor $\operatorname{size}^{2,9}$. Untreated VS will potentially destroy functional hearing within a couple of years ${ }^{10}$.

Surgical VS treatment not only aims on removing the tumor but also preserving facial nerve function and auditory function - the latter, if still present. Numerous factors influence hearing outcome. Besides tumor size and preoperative hearing status $s^{6,11-15}$, hearing preservation is reported to be more likely if the tumor arises from the SVN ${ }^{11,16,17}$. This is thought to be due to a closer anatomical relationship between the IVN, the cochlear nerve and the internal auditory artery. Dissecting a tumor arising from the IVN may involve a greater risk for compromising blood supply and traumatizing the cochlear nerve directly ${ }^{16,17}$. Knowledge about the nerve of

${ }^{1}$ Department of Otorhinolaryngology, Head and Neck Surgery, University Hospital Halle (Saale), Martin Luther University Halle-Wittenberg, Halle (Saale), Germany. ${ }^{2}$ Department of Neurosurgery, University Hospital Halle (Saale), Martin Luther University Halle-Wittenberg, Halle (Saale), Germany. ${ }^{3}$ Universitäts-HNO-Klinik, Ernst-Grube-Str. 40, 06120 Halle (Saale), Germany. ${ }^{\circledR}$ email: torsten.rahne@uk-halle.de 
origin could assist the surgeon in the intraoperative assessment of pathological anatomy, and facilitate better tumor removal. Preoperative differentiation of the nerve of origin may influence the decision on treatment, and the selection of optimal treatment and counselling modalities ${ }^{18}$.

Vestibular function is dependent on IVN and SVN integrity. However, it is not in the focus of a recent guideline on the diagnosis and treatment of $\mathrm{VS}^{2}$. It has been shown that vestibular function test results for the semicircular canals and the otoliths potentially allow to differentiate between IVN and SVN functional deficits and thus the respective VS origin ${ }^{18,19}$. Semicircular canal function can be measured with video head-impulse testing (vHIT) ${ }^{20,21}$. Based on the vestibular-ocular reflex, head and eye velocities are recorded simultaneously during and after a head impulse. Based on which of the canals are showing pathological results, an allocation to IVN and SVN deficits is possible $e^{22-25}$.

The cervical vestibular-evoked myogenic potential (cVEMP) test mostly evaluates saccular function while the ocular vestibular-evoked myogenic potential (oVEMP) test assesses mostly utricular function ${ }^{26-29}$. Since SVN and IVN integrity are prerequisites for assessment of otolith function, cVEMP and oVEMP recordings could conceivably differentiate between SVN- and IVN-mediated disturbance ${ }^{25,29-33}$.

Preoperative VEMP studies alone predict the nerve of origin inconsistently ${ }^{34}$, and a combination with caloric results did not show a correlation ${ }^{35}$. In a preliminary study, we reported a scoring algorithm integrating vHIT, cVEMP and oVEMP results to differentiate between tumor origin from IVN or SVN confirmed by intraoperative findings ${ }^{18}$. In that study, the score predicted tumor origin correctly in four of five consecutive VS patients.

This study aims on applying the preoperative scoring algorithm based on vHIT, cVEMP and oVEMP results in a large cohort of consecutive patients with VS surgery including those five of the initial study ${ }^{18}$ and to compare the predictions with the intraoperative surgically assessed tumor origin.

\section{Methods}

Study design and population. In a tertiary interdisciplinary referral centre at a university hospital, we conducted a double-blind clinical diagnostic study. We assessed eligibility of all consecutive adult patients undergoing primary surgery for VS treatment between January 2016 and March 2020. The study was approved by Ethical committee of the Medical Faculty of the Martin-Luther-University Halle-Wittenberg (approval number 2017-103) and conducted in accordance with the Declaration of Helsinki. Informed consent was obtained from all participants for being included in the study.

Preoperative testing. Patients underwent audiological and vestibular tests 1 day before surgery as part of the routine clinical protocol. Routine otomicroscopic evaluation of the external ear canals and the tympanic membranes were performed prior to collecting pure-tone audiogram including air-conduction hearing thresholds for frequencies of $0.125-8 \mathrm{kHz}$; and bone-conduction hearing thresholds for frequencies of $0.25-6 \mathrm{kHz}$. Word recognition score (WRS) was determined using the Freiburg speech test. Lists of monosyllabic words and multisyllabic numbers at various sound pressure levels (SPL) were presented with headphones. If a WRS of $100 \%$ was not achieved at $65 \mathrm{~dB}$ SPL, sound pressure was increased to a maximum of $100 \mathrm{~dB}$ SPL and the maximum percentage of monosyllables understood $\left(\mathrm{WRS}_{\max }\right.$ ) was determined. Hearing was classified according to ${ }^{12,36}$, adapted for German speech recognition tests ${ }^{37}$. Audiological assessments were conducted using an AT900 audiometer (Auritec, Hamburg, Germany).

To evaluate the vestibulo-ocular reflex (VOR), the head-impulse test was performed with a vHIT system (GN Otometrics, Taastrup, Denmark) according to Rahne et al. ${ }^{18}$. A light (60 g) and high-speed $(250 \mathrm{~Hz})$ eye camera with a built-in calibration laser was connected to the patient, enabling identification of overt and covert saccades. The head was quickly and unpredictably turned through $10^{\circ}-20^{\circ}$ angles in the horizontal, the LARP or RALP planes, to facilitate testing of the corresponding semicircular canals (LARP: left anterior-right posterior; RALP: right anterior-left posterior). We evaluated the absolute mean VOR gain between eye and head movement of the affected and non-affected sides. The appearance of covert and overt saccades after head impulses was analysed subjectively ${ }^{18}$. Artifacts due to methodological errors were avoided during the vHIT recording. Two experienced investigators analysed the recordings. If less than five clear covert or overt saccades could be discerned the respective SCC was labelled as free of saccades.

Air-conducted cVEMP were measured using the Eclipse Platform (Interacoustics, Copenhagen, Denmark), with surface electrodes placed on the upper half of the ipsilateral sternocleidomastoid muscles, a reference electrode on the mastoid, and the ground electrode on the forehead. An insert earphone was used to apply monaural acoustic stimulation as tone bursts of $500 \mathrm{~Hz}$ (rise/fall time, $2 \mathrm{~ms}$; plateau time, $1 \mathrm{~ms} ; 100 \mathrm{~dB} \mathrm{nHL}$ ) ${ }^{18}$,

38. The stimulus rate was $5.1 \mathrm{~Hz}$. For every presentation, at least 200 stimuli were averaged. After excluding a conductive hearing loss, the patients took a seated position and were instructed to rotate their heads toward the non-stimulated ear. Measuring the electromyogram (EMG) amplitude and delivering acoustic feedback to the patient maintained constant muscle tension. The first positive-negative peak (p13-n23) of the averaged EMG was defined as the cVEMP amplitude.

Air-conducted oVEMP were measured by placing the electrodes directly underneath the patients' contralateral eyes, and applying acoustic stimuli to the ipsilateral ear. The ground electrode was placed on the forehead. The patients were asked to look maximally upwards while hearing the tones. The first negative-positive peak (n10-p15) of the averaged EMG was defined as the oVEMP amplitude ${ }^{18}$. Other stimulation and recording parameters were as described above for the cVEMP recordings.

We calculated the asymmetry ratios (AR) of the cVEMP and oVEMP amplitudes $\left(\mathrm{AR}_{\mathrm{CVEMP}}\right.$ and $\mathrm{AR}_{\text {oVEMP }}$ respectively) between the tumor-affected side and the non-affected side, using the ipsilateral (IA) and contralateral (CA) amplitudes at $100 \mathrm{~dB}$ nHL: $\mathrm{AR}=(\mathrm{IA}-\mathrm{CA}) /(\mathrm{IA}+\mathrm{CA})^{39}$. 


\begin{tabular}{|l|l|l|l|l|}
\hline \multirow{2}{*}{ Assessment } & \multirow{3}{*}{ Objective } & \multirow{2}{*}{ Threshold } & \multicolumn{3}{|l|}{ Scoring } \\
\cline { 4 - 6 } vHIT & IVN & SVN \\
\hline \multirow{2}{*}{ Anterior semicircular canal } & Gain & $<0.7$ & 0 & 1 \\
\cline { 2 - 6 } & Saccades & Present & 0 & 1 \\
\hline \multirow{2}{*}{ Lateral semicircular canal } & Gain & $<0.8$ & 0 & 1 \\
\cline { 2 - 6 } & Saccades & Present & 0 & 1 \\
\hline \multirow{2}{*}{ Posterior semicircular canal } & Gain & $<0.7$ & 2 & 0 \\
\cline { 2 - 6 } & Saccades & Present & 2 & 0 \\
\hline VEMP & $\mathrm{AR}_{\text {cVEMP }}$ & $<-0.36$ & 4 & 0 \\
\hline Cervical & $\mathrm{AR}_{\text {oVEMP }}$ & $<-0.36$ & 0 & 4 \\
\hline Ocular & \multicolumn{5}{|l}{} \\
\hline Maximum score: & \multicolumn{5}{|l}{} \\
\hline
\end{tabular}

Table 1. Scoring thresholds for determination of the most affected nerve of origin.

The nerve of origin was predicted by using the scoring system according to Rahne et al. ${ }^{18}$ (Table 1). A gain or asymmetry ratio smaller than the threshold levels was defined as pathologic. An allocation to either the anterior or lateral semicircular canals contributed one point to the SVN, while a pathological gain allocated to the posterior semicircular canal contributed two points to the IVN. Saccade occurrence allocated to either the anterior or lateral semicircular canals contributed one point each to the SVN, while saccade occurrence allocated to the posterior semicircular canal contributed two points to the IVN. Pathological cVEMP or oVEMP asymmetry ratios contributed four points to the IVN or SVN, respectively. Due to the increased weight for the posterior semicircular gain, the scoring was balanced between both nerve branches, with the potential to achieve a total score of eight.

Surgery. Prior to surgery tumors were categorized according to the Koos classification ${ }^{13}{ }^{40}$. With patients in the supine position and under total intravenous anaesthesia, tumor resection was performed via a retrosigmoid approach using facial and cochlear nerve monitoring. All operations were performed with the goal of preserving facial, cochlear, and remaining vestibular nerve function. During surgery, at least one experienced neurosurgeon (CS) who was blinded to the diagnostic results identified the nerve of VS origin. Criteria were adherence of the schwannoma to either vestibular nerves within the internal auditory canal and difficulties of dissection tumor from the respective vestibular portions, or in schwannomas with the fundus free of tumor, visual identification of the tumor origin. If the fundus is obliterated the position of the transverse crest at the fundus as localised with the rectangular nerve hook is helpful. In those tumors arising from the superior vestibular nerve the transverse crest is identified at a more caudal position due to the tumor growth pattern and vice versa. In isolated cases an endoscope was used. If the tumor's origin could not be unambiguously determined, then the most likely nerve of origin was selected and reported to the data evaluation team. The diagnostic investigators were blinded to intraoperative findings.

Study outcomes and statistical analysis. The primary outcomes were the sensitivity and specificity of prediction of intraoperative finding by a preoperative score. The Koos grade was recorded and used for stratification. According to Rahne et al. ${ }^{18}$, the scores for prediction of nerve origin were calculated based on amplitudes of the cVEMPs and oVEMPs, as well as the vHIT gain and occurrence of saccades. The nerve with the higher score was assumed to be most affected and was, thus, the presumptive nerve of origin.

The confusion matrix was calculated for IVN and SVN including sensitivity, specificity, positive and negative predictive values and Matthews correlation coefficient ${ }^{41}$. A multiple logistic regression was calculated to predict tumor origin based on vHIT gains, vHIT saccades, cVEMP AR and oVEMP AR. Receiver operating characteristic (ROC) curve analysis was performed for the primary outcome parameters including calculation of true positive, true negative, false negative and true negative rates for both, IVN and SVN nerve origin. Sensitivity and specificity were computed. Based on the results an optimized combination of predictive outcome was hypothesized using Youden's index (true positive rate plus false negative rate ${ }^{42}$ ). The index $J$ is an ROC parameter $(J=$ sensitivity + specificity -1$)$ with values between 0 and 1 . A large index graphically represents a large distance of the ROC curve from chance level (diagonal line) that is equivalent to a good discrimination performance. The maximum value of the index was used as criterion for selecting the optimum cut-off values of the diagnostic tests. All statistical analyses was performed using the SPSS software version 25 (IBM, Ehningen, Germany).

\section{Results}

A total of 93 patients undergoing surgery for VS treatment were included in the study (mean age 51.6 years, range 18-77 years; 28 male, 65 female). Demographic details are shown in Table 2. According to the Koos classification, tumors were diagnosed as stage I [13 patients (14\%)], II [28 patients (30\%)], III [31 patients (33\%)], or IV [21 patients (23\%)]. All included patients underwent surgery for tumor removal. After surgery, 13 patients were excluded from further analysis (see Fig. 1). Complete resection was achieved in all remaining cases. 


\begin{tabular}{|c|c|c|c|c|c|c|c|c|c|c|}
\hline \multirow[b]{2}{*}{ Koos grade } & \multirow[b]{2}{*}{$\mathbf{n}$} & \multirow[b]{2}{*}{ Male/female (n) } & \multirow[b]{2}{*}{ Mean age (SD)/years } & \multirow[b]{2}{*}{ Right/left (n) } & \multicolumn{2}{|c|}{ 4PTA (SD)/dB HL } & \multirow{2}{*}{$\begin{array}{l}\text { Mean } \\
\text { WRS }_{65}(\mathrm{SD})\end{array}$} & \multirow{2}{*}{\begin{tabular}{|l|} 
Mean \\
WRS $_{\max }(\mathrm{SD})$ \\
\end{tabular}} & \multicolumn{2}{|c|}{$\begin{array}{l}\text { Median hearing class } \\
(95 \% \mathrm{CI})\end{array}$} \\
\hline & & & & & Ipsilateral & Contralateral & & & AAO-HNS ${ }^{\mathrm{a}}$ & GR \\
\hline Total & 80 & $26 / 54$ & $51.5(12.7)$ & $42 / 38$ & $49.4(31.7)$ & $17.1(10.7)$ & $49(39)$ & $70(36)$ & $2(2.1 ; 2.6)$ & $\begin{array}{l}2(2.1 ; \\
2.6)\end{array}$ \\
\hline I & 12 & $3 / 9$ & $48.1(14.4)$ & $8 / 4$ & $35.3(25.1)$ & $15.5(10.8)$ & $59(41)$ & $81(24)$ & $1(1.1 ; 2.8)$ & $\begin{array}{l}1(1.1 \\
2.4)\end{array}$ \\
\hline II & 26 & $6 / 20$ & $53.9(13.1)$ & $14 / 12$ & $45.8(27.3)$ & $18.8(10.2)$ & $53(37)$ & $79(29)$ & $2(1.8 ; 2.6)$ & $\begin{array}{l}2(1.8 ; \\
2.6)\end{array}$ \\
\hline III & 25 & $10 / 15$ & $52.1(11.2)$ & $12 / 13$ & $52.5(32.7)$ & $16.6(11.9)$ & $45(39)$ & $69(36)$ & $2(1.9 ; 2.9)$ & $\begin{array}{l}2(1.8 ; \\
2.8)\end{array}$ \\
\hline IV & 17 & $7 / 10$ & $49.1(13.3)$ & $8 / 9$ & $60.3(38.1)$ & $16.6(10.3)$ & $43(42)$ & $53(46)$ & $3(2.1 ; 3.4)$ & $\begin{array}{l}3(2.1 ; \\
3.9)\end{array}$ \\
\hline
\end{tabular}

Table 2. Demographic and audiological details of tumor patients. ${ }^{\mathrm{a}} 1=\mathrm{A}, 2=\mathrm{B}, 3=\mathrm{C}, 4=\mathrm{D} ; \mathrm{SD}$ standard deviation, dB HL decibels hearing level, GR Gardner \& Robertson, $4 P T A$ pure tone average at $0.5,1,2,4 \mathrm{kHz}$, $W R S$ word recognition score at $65 \mathrm{~dB}$ sound pressure level, $W R S_{\text {max }}$ maximum WRS.

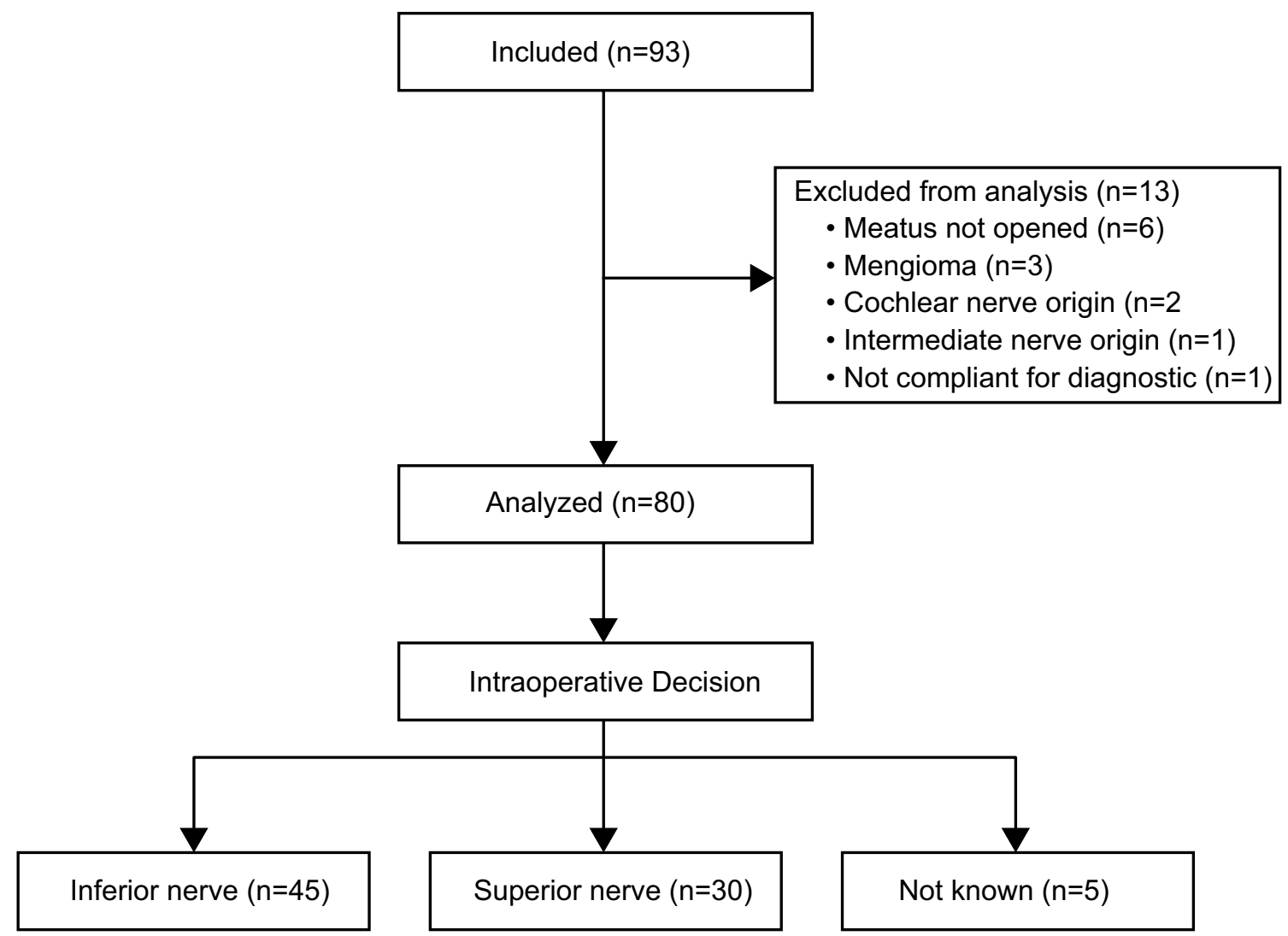

Figure 1. Flow chart of included excluded and analysed patients, as well as distribution of intraoperative decisions regarding nerve of origin.

Figure 1 shows the intraoperative diagnosis of 45 (56\%) patients with an IVN nerve origin and 30 (38\%) patients with an SVN origin. In five (6\%) patients, the surgeon could not make a clear decision. Applying the scoring system of Rahne et al. ${ }^{18}$, the preoperatively determined nerve of tumor origin was the SVN in $23(29 \%)$ patients, the IVN in 40 (50\%) patients, and $17(21 \%)$ decisions were indifferent.

Table 3 shows the confusion matrix for both nerves of origin. For the prediction of IVN origin sensitivity was $73 \%$ and specificity was $80 \%$. For the prediction of SVN origin sensitivity was $60 \%$ and specificity was $90 \%$. Matthews's correlation coefficients were 0.529 for IVN and 0.535 for SVN. Figure 2 shows the sensitivity and specificity stratified by the Koos grade. Sensitivity and specificity did not vary very much across the subgroups.

Table 4 shows the logistic regression analysis of SVN nerve of origin prediction. A significant regression was found for the compound model $\left[\chi^{2}(8)=46.0, p<0.001\right]$ with a Nagelkerke $R^{2}$ of 0.625 which reflects a very strong effect. vHIT gains and occurrence of saccades for the anterior and lateral SCC as well as cVEMP ARs were significant predictors of the tumor origin. 


\begin{tabular}{|c|c|c|c|c|c|}
\hline \multicolumn{6}{|c|}{ Superior vestibular nerve (SVN) } \\
\hline & & \multicolumn{2}{|c|}{ Surgical finding } & \multicolumn{2}{|c|}{ Predictive value } \\
\hline & & SVN & not SVN & Positive & Negative \\
\hline \multirow{4}{*}{ Predicted origin } & SVN & 18 & 5 & 0.78 & \\
\hline & Not SVN & 12 & 45 & & 0.71 \\
\hline & Sensitivity & 0.60 & & & \\
\hline & Specificity & & 0.90 & & \\
\hline \multicolumn{6}{|c|}{\begin{tabular}{|l|} 
Inferior vestibular nerve (IVN) \\
\end{tabular}} \\
\hline & & \multicolumn{2}{|c|}{ Surgical finding } & \multicolumn{2}{|c|}{ Predictive value } \\
\hline & & IVN & not IVN & Positive & Negative \\
\hline \multirow{4}{*}{ Predicted origin } & IVN & 33 & 7 & 0.83 & \\
\hline & Not IVN & 12 & 28 & & 0.46 \\
\hline & Sensitivity & 0.73 & & & \\
\hline & Specificity & & 0.80 & & \\
\hline
\end{tabular}

Table 3. Confusion matrix.



Figure 2. Sensitivity and specificity of the nerve of origin prediction by the scoring system stratified by Koos grade.

\begin{tabular}{|c|c|c|c|c|c|c|c|c|}
\hline Predictor & $\beta$ & Standard error of $\beta$ & Wald's $\chi^{2}$ & $d f$ & $p$ & $e^{\beta}$ & $95 \% \mathrm{c}$ & of $e^{\beta}$ \\
\hline Constant term & -1.814 & 2.331 & 0.605 & 1 & 0.437 & NA & NA & NA \\
\hline vHIT gain anterior SCC & 6.817 & 3.455 & 3.893 & 1 & 0.048 & 913.283 & 1.047 & $797,013.613$ \\
\hline vHIT gain lateral SCC & -5.922 & 2.767 & 4.582 & 1 & 0.032 & 0.003 & 0.000 & 0.607 \\
\hline $\begin{array}{l}\text { vHIT gain posterior } \\
\text { SCC }\end{array}$ & 3.021 & 1.811 & 2.781 & 1 & 0.095 & 20.508 & 0.589 & 714.075 \\
\hline $\begin{array}{l}\text { vHIT Saccades anterior } \\
\text { SCC }\end{array}$ & 2.817 & 1.143 & 6.073 & 1 & 0.014 & 16.719 & 1.780 & 157.060 \\
\hline $\begin{array}{l}\text { vHIT Saccades lateral } \\
\text { SCC }\end{array}$ & -2.855 & 0.886 & 10.376 & 1 & 0.001 & 0.058 & 0.633 & 24.198 \\
\hline $\begin{array}{l}\text { vHIT Saccades posterior } \\
\text { SCC }\end{array}$ & 1.364 & 0.930 & 2.154 & 1 & 0.142 & 3.913 & 0.010 & 0.327 \\
\hline cVEMP AR & 3.064 & 1.311 & 5.460 & 1 & 0.019 & 21.407 & 1.639 & 279.645 \\
\hline oVEMP AR & -1.168 & 0.923 & 1.603 & 1 & 0.205 & 0.311 & 0.051 & 1.897 \\
\hline
\end{tabular}

Table 4. Logistic regression analysis of superior nerve of origin prediction. AR asymmetry ratio, $c V E M P$ cervical vestibular evoked potentials, NA not applicable, oVEMP ocular vestibular evoked potentials, SCC semicircular canal. 


\section{ROC curves of vHIT gain}
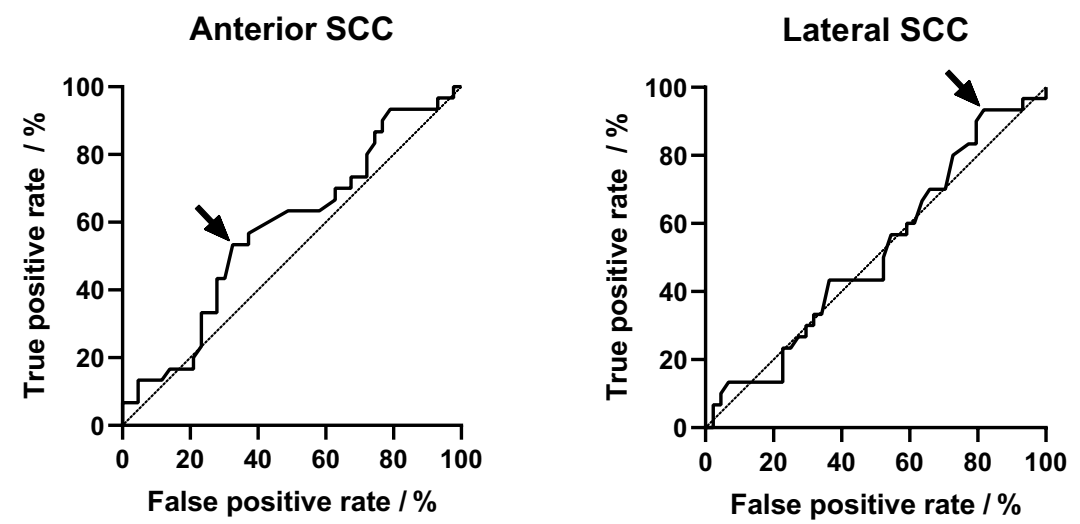

Posterior SCC



\section{ROC curves of vHIT asymmetry ratio}

\section{Anterior SCC}



Lateral SCC

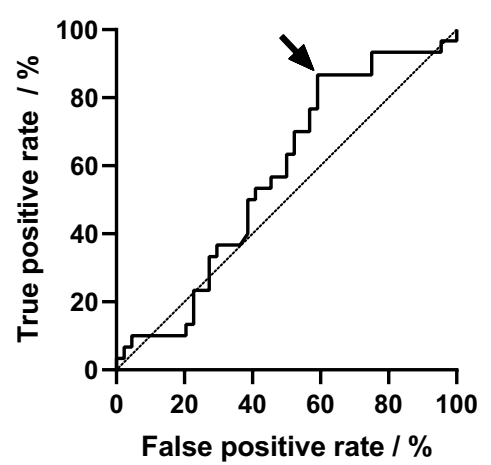

Posterior SCC

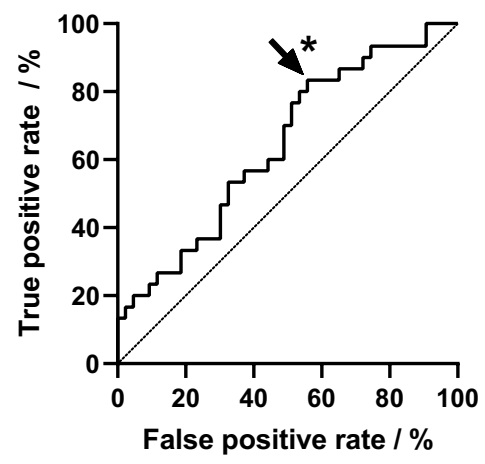

Figure 3. ROC curves for nerve of origin analysis based on vHIT gain and asymmetry ratio measures. The diagonal marks random classification. Asterisks $\left({ }^{*}\right)$ mark significant areas under curve $(\mathrm{p}<0.05)$. Arrows mark the optimized cut-off value.

Figure 3 shows the ROC curves for the vHIT gain and asymmetry ratios for all semicircular canals. Figure 4 shows the ROC curves for the CVEMP and oVEMP amplitudes and the respective asymmetry ratios. The figures show the test characteristics for the used vHIT and c/oVEMP thresholds. For the vHIT gain and asymmetry ratio of the posterior SCC, cVEMP amplitude and cVEMP asymmetry ratio, the area under the curves revealed significant classification $(p<0.05)$. Based on the trade-off between sensitivity and specificity, i.e., a maximized Youden's index, optimized cut-off values were identified as -0.32 for cVEMP and -0.11 for oVEMP asymmetry ratios. Optimized vHIT gain thresholds would be 0.77 (anterior SCC), 0.84 (lateral SCC) and 0.80 (posterior SCC).

The scoring system was optimized by including vHIT gain and VEMP asymmetry ratio. Applying the improved scoring system based on the optimized cut-off values, the preoperatively predicted nerve of tumor origin was the SVN in 27 (34\%) patients, and the IVN in $42(52 \%)$ patients, while 11 (14\%) decisions were indifferent.

Table 5 shows the confusion matrix for both nerves of origin after cut-off value optimization. For the prediction of IVN, origin sensitivity was $76 \%$ and specificity was $77 \%$. For the prediction of SVN origin, sensitivity was $70 \%$ and specificity was $88 \%$. Matthews's correlation coefficient was 0.523 for IVN and 0.594 for SVN.

\section{Discussion}

In this study, VEMP and vHIT measurements for assessing vestibular receptor function were used to predict the nerve of origin for VS in a large number of patients. The results show prediction of the nerve of tumor origin diagnosed intraoperatively well above chance level. Sensitivity was larger for IVN tumors than for SVN tumors. Vice versa, specificity was larger for SVN tumors.

The prediction was based on the scoring system by Rahne et al. ${ }^{18}$ including vHIT amplitudes and saccades as well as AR of cVEMP and oVEMP amplitudes comparing the affected side to the non-affected side. Thus, several factors could have limited the predictability.

VS could potentially affect both nerves, as observed in the current dataset. Thus, to determine the nerve of origin, we hypothesized that the nerve with the highest score is the nerve of tumor origin. In the majority of cases, an experienced surgeon was able to determine the nerve of origin intraoperatively. Therefore, the risk of intraoperative misjudgement was considered very low in our study. 


\section{ROC curves of cVEMP}

\section{Amplitude}

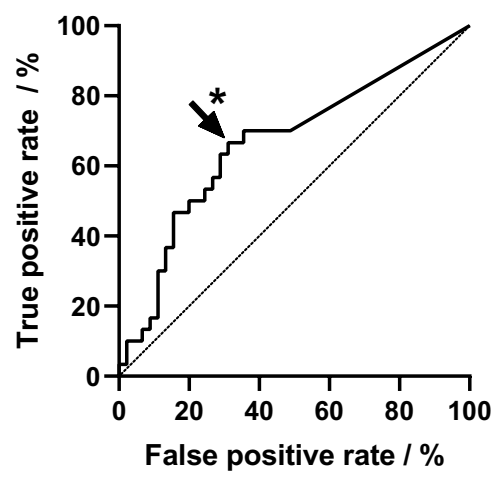

ROC curves of oVEMP

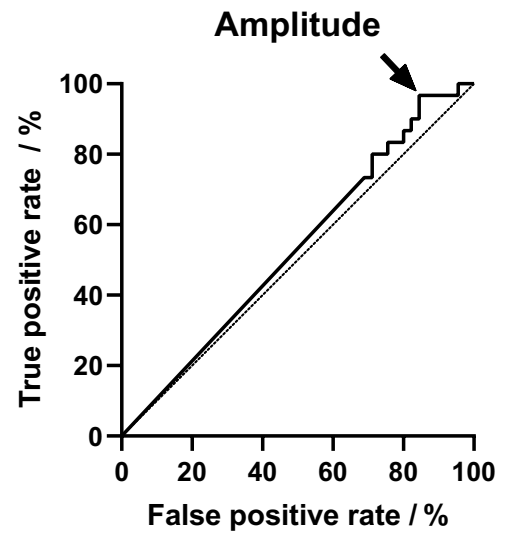

\section{Asymmetry ratio}

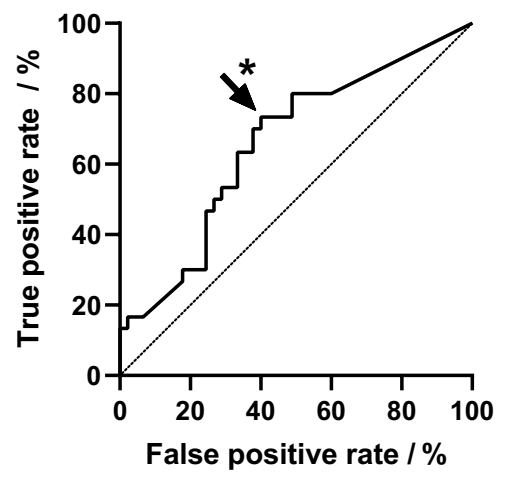

Asymmetry ratio

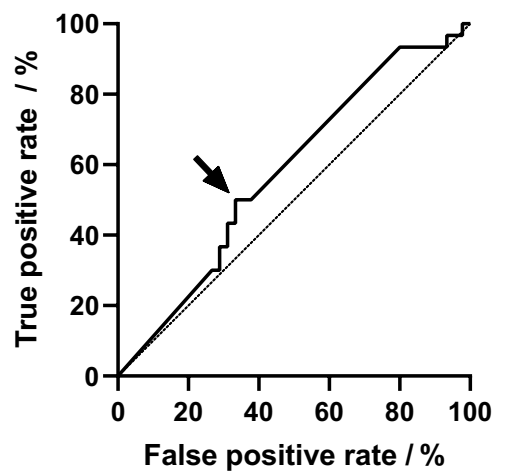

Figure 4. ROC curves for nerve of origin analysis based on cVEMP and oVEMP amplitude and asymmetry ratio measures. The diagonal marks random classification. Asterisks $\left(^{*}\right)$ mark significant areas under curve $(\mathrm{p}<0.05)$. Arrows mark the optimized decision value.

\begin{tabular}{|c|c|c|c|c|c|}
\hline \multicolumn{6}{|c|}{ Superior vestibular nerve (SVN) } \\
\hline & & \multicolumn{2}{|c|}{ Surgical finding } & \multicolumn{2}{|c|}{ Predictive value } \\
\hline & & SVN & not SVN & Positive & Negative \\
\hline \multirow{4}{*}{ Predicted origin } & SVN & 21 & 6 & 0.78 & \\
\hline & Not SVN & 9 & 44 & & 0.68 \\
\hline & Sensitivity & 0.70 & & & \\
\hline & Specificity & & 0.88 & & \\
\hline \multicolumn{6}{|c|}{ Inferior vestibular nerve (IVN) } \\
\hline & & \multicolumn{2}{|c|}{ Surgical finding } & \multicolumn{2}{|c|}{ Predictive value } \\
\hline & & IVN & not IVN & Positive & Negative \\
\hline \multirow{4}{*}{ Predicted origin } & IVN & 34 & 8 & 0.81 & \\
\hline & Not IVN & 11 & 27 & & 0.44 \\
\hline & Sensitivity & 0.76 & & & \\
\hline & Specificity & & 0.77 & & \\
\hline
\end{tabular}

Table 5. Confusion matrix based on optimized decision criteria. 
Despite VS induced hearing loss is widely independent of the tumor size ${ }^{43}$, the disturbance of vestibular nerve function increases with tumor size ${ }^{9}$. Large tumors are more likely to affect both, IVN and SVN and thus the results of VHIT and VEMP. Thus, tumor size seems to be a limiting factor for the prediction power. In the current study, the majority of tumors were categorized to Koos stages 2 and 3. As medium-sized tumors demonstrate the highest gain asymmetry ${ }^{44}$, we would expect a slightly larger prediction power, if only medium sized tumors were assessed. However, sensitivity and specificity did not vary very much across the subgroups (Fig. 2).

It is also possible for the cochlear or intermediate nerve to be the nerve of origin of a tumor in the cerebellopontine angle, such that the presently evaluated method could be misleading in a minority of cases. However, the main objective in deriving a preoperative prediction of the nerve of origin was to gather information about the risk for hearing loss, which would be mitigated, if the tumor origin was in the SVN ${ }^{17}$. That prediction would be irrelevant for cases of VS originating in the cochlear nerve. In the current study, such patients were excluded to focus on the prediction method on separating IVN and SVN tumors only.

Hearing preservation is dependent on the tumor size and on other anatomical factors as position of the VS in relation to the IAC or enlargement of the IAC. Also excreted exosomes from the tumors may be cochleotoxic independent or additionally to the nerve of tumor origin ${ }^{45}$, ${ }^{46}$. Nevertheless, determining the tumor origin prior to surgery may lead to improved predictions for hearing preservation and could thus become useful in counselling patients.

There is still some variation regarding the critical values (cut-off values) used to classify reduced vestibular function as pathologic. The criteria applied in our presently evaluated scoring system depend on the use of generally accepted cut-off values for vHIT ${ }^{47,48}$ and VEMP ${ }^{49,50}$. The present results allow for separately analysing the applied outcome parameters to optimize the cut-off values to increase sensitivity and specificity.

Our results reveal a rather small discrimination power between IVN and SVN tumors by using vHIT amplitudes alone (Fig. 3). The best discrimination power was observed if the posterior SCC was assessed by vHIT. Despite vHIT overall shows a large sensitivity in diagnosing $\mathrm{VS}^{44}$, prediction of tumor origin with vHIT alone would not be precise enough.

A good prediction power was observed, when cVEMP responses were compared between the affected and unaffected sides (Fig. 4). The discriminatory power based on the oVEMP responses, however, was very small. Here, the use of more effective stimulation methods, e.g., a powerful bone vibrator may reduce the large number of patients with no air-conduction driven oVEMP responses. Since VEMP responses are reduced specifically in the affected nerve, a combined analysis based on cVEMP and (BC) oVEMP responses might provide a high sensitivity for the prediction of nerve of origin. Sensitivity of oVEMP could potentially be improved by including bone-conduction stimulation.

The prediction power of the scoring system might also be limited by the age dependence of VEMP responses. VEMP test in the elderly is consider unreliable due to loss of hair cells of saccular and utricular macula in subjects aged $>60$ years. Therefore, in those patients absent VEMP responses cannot clearly be differentiated caused by either aging or pathological effect. We hypothesize that the introduced scoring system would be more reliable if applied only to patients aged $<60$ years.

Since both vHIT and VEMP results gradually contribute to the SVN and IVN assessment, the scoring system provides a compound rating method that should reduce false negatives via added redundancy. Cut-off values categorize vestibular sensor function dichotomously and specifically for each sensor ${ }^{21,32,51}$ and were optimized by the results. The optimized values found were rather close to the previously used values. The sensitivity was improved for SVN and IVN tumors while the specificity was still large (Table 4).

\section{Conclusions}

The results reported here suggest that a prediction of the nerve of origin of VS is possible using the applied scoring system with a high agreement between preoperative and intraoperative findings. It is proposed that small VS tumors correlate with disturbance or reduced integrity of either the SVN or IVN, as reflected by pathological function of the semicircular canals or otoliths, respectively. Larger tumors are likely to also affect the other vestibular nerve branch and the intermediate or cochlear nerves as well. The assessments methods are commonly available in skull base centres with neurotological diagnostics. Its application could potentially improve prediction of tumour origin and its implication for hearing preservation.

Received: 1 February 2021; Accepted: 30 March 2021

Published online: 21 April 2021

\section{References}

1. Merchant, S. N. \& Nadol, J. B. Cochleovestibular Schwannoma. In Schuknecht's Pathology of the Ear 3rd edn (eds Merchant, S. N. \& Nadol, J. B.) 492-508 (People’s Medical Publishing, 2010).

2. Goldbrunner, R. et al. EANO guideline on the diagnosis and treatment of vestibular schwannoma. Neuro Oncol. 20, 20 (2019).

3. Møller, M. N., Hansen, S. \& Caye-Thomasen, P. Peripheral vestibular system disease in vestibular schwannomas: A human temporal bone study. Otol. Neurotol. 36, 1547-1553. https://doi.org/10.1097/MAO.0000000000000846 (2015).

4. Xenellis, J. E. \& Linthicum, F. H. On the myth of the glial/schwann junction (Obersteiner-Redlich zone): Origin of vestibular nerve schwannomas. Otol. Neurotol. 24, 1 (2003).

5. He, Y.-B., Yu, C.-J., Ji, H.-M., Qu, Y.-M. \& Chen, N. Significance of vestibular testing on distinguishing the nerve of origin for vestibular schwannoma and predicting the preservation of Hearing. Chin. Med. J. 129, 799-803. https://doi.org/10.4103/0366-6999. 178958 (2016).

6. Khrais, T., Romano, G. \& Sanna, M. Nerve origin of vestibular schwannoma: A prospective study. J. Laryngol. Otol. 122, $128-131$. https://doi.org/10.1017/S0022215107001028 (2008).

7. Roosli, C., Linthicum, F. H., Cureoglu, S. \& Merchant, S. N. What is the site of origin of cochleovestibular schwannomas?. Audiol. Neuro-otol. 17, 121-125. https://doi.org/10.1159/000331394 (2012). 
8. Plontke, S. K. et al. How much cochlea do you need for cochlear implantation?. Otol. Neurotol. https://doi.org/10.1097/MAO. $0000000000002614(2020)$.

9. Andersen, J. F. et al. Predictors of vertigo in patients with untreated vestibular schwannoma. Otol. Neurotol. 36, 647-652. https:// doi.org/10.1097/MAO.0000000000000668 (2015).

10. Tveiten, O. V. et al. Long-term auditory symptoms in patients with sporadic vestibular schwannoma: An international crosssectional study. Neurosurgery 77, 218-227. https://doi.org/10.1227/NEU.0000000000000760 (2015) ((discussion 227)).

11. Brackmann, D. E. et al. Prognostic factors for hearing preservation in vestibular schwannoma surgery. Am. J. Otol. 21, 417-424 (2000).

12. Gardner, G. \& Robertson, J. H. Hearing preservation in unilateral acoustic neuroma surgery. Ann. Otol. Rhinol. Laryngol. 97, 55-66. https://doi.org/10.1177/000348948809700110 (1988).

13. Koos, W. T., Day, J. D., Matula, C. \& Levy, D. I. Neurotopographic considerations in the microsurgical treatment of small acoustic neurinomas. J. Neurosurg. 88, 506-512. https://doi.org/10.3171/jns.1998.88.3.0506 (1998).

14. Samii, M., Gerganov, V. \& Samii, A. Improved preservation of hearing and facial nerve function in vestibular schwannoma surgery via the retrosigmoid approach in a series of 200 patients. J. Neurosurg. 105, 527-535. https://doi.org/10.3171/jns.2006.105.4.527 (2006).

15. Satar, B., Yetiser, S. \& Ozkaptan, Y. Impact of tumor size on hearing outcome and facial function with the middle fossa approach for acoustic neuroma: A meta-analytic study. Acta Oto-laryngol. 123, 499-505. https://doi.org/10.1080/00016480310000566a (2003).

16. Jacob, A. et al. Nerve of origin, tumor size, hearing preservation, and facial nerve outcomes in 359 vestibular schwannoma resections at a tertiary care academic center. Laryngoscope 117, 2087-2092. https://doi.org/10.1097/MLG.0b013e3181453a07 (2007).

17. Rachinger, J. et al. Tumor origin and hearing preservation in vestibular schwannoma surgery. J. Neurosurg. 115, 900-905. https:// doi.org/10.3171/2011.7.JNS102092 (2011).

18. Rahne, T., Plößl, S., Plontke, S. K. \& Strauss, C. Preoperative determination of nerve of origin in patients with vestibular schwannoma. HNO 66, 16-21. https://doi.org/10.1007/s00106-017-0416-y (2018).

19. von Kirschbaum, C. \& Gürkov, R. Audiovestibular function deficits in vestibular schwannoma. BioMed Res. Int. 2016, 4980562. https://doi.org/10.1155/2016/4980562 (2016).

20. MacDougall, H. G., McGarvie, L. A., Halmagyi, G. M., Curthoys, I. S. \& Weber, K. P. The video Head Impulse Test (vHIT) detects vertical semicircular canal dysfunction. PLoS One 8, e61488. https://doi.org/10.1371/journal.pone.0061488 (2013).

21. Weber, K. P., MacDougall, H. G., Halmagyi, G. M. \& Curthoys, I. S. Impulsive testing of semicircular-canal function using videooculography. Ann. New York Acad. Sci. 1164, 486-491. https://doi.org/10.1111/j.1749-6632.2008.03730.x (2009).

22. Batuecas-Caletrio, A., Santa Cruz-Ruiz, S., Muñoz-Herrera, A. \& Perez-Fernandez, N. The map of dizziness in vestibular schwannoma. Laryngoscope 125, 2784-2789. https://doi.org/10.1002/lary.25402 (2015).

23. Blödow, A., Blödow, J., Bloching, M. B., Helbig, R. \& Walther, L. E. Horizontal VOR function shows frequency dynamics in vestibular schwannoma. Eur. Arch. Oto-Rhino-Laryngol. 272, 2143-2148. https://doi.org/10.1007/s00405-014-3042-2 (2015).

24. Constanzo, F., Sens, P., Teixeira, B. C. D. A. \& Ramina, R. Video head impulse test to preoperatively identify the nerve of origin of vestibular Schwannomas. Oper. Neurosurg. (Hagerstown, Md.) 16, 319-325. https://doi.org/10.1093/ons/opy103 (2019).

25. Suzuki, M. et al. Analysis of vestibular testing in patients with vestibular schwannoma based on the nerve of origin, the localization, and the size of the tumor. Otol. Neurotol. 29, 1029-1033. https://doi.org/10.1097/MAO.0b013e3181845854 (2008).

26. Chiarovano, E., Darlington, C., Vidal, P.-P., Lamas, G. \& de Waele, C. The role of cervical and ocular vestibular evoked myogenic potentials in the assessment of patients with vestibular schwannomas. PLoS One 9, e105026. https://doi.org/10.1371/journal.pone. 0105026 (2014).

27. Halmagyi, G. M. \& Curthoys, I. S. Clinical testing of otolith function. Ann. N. Y. Acad. Sci. 871, 195-204. https://doi.org/10.1111/j. 1749-6632.1999.tb09185.x (1999).

28. Piras, G., Brandolini, C., Castellucci, A. \& Modugno, G. C. Ocular vestibular evoked myogenic potentials in patients with acoustic neuroma. Eur. Arch. Oto-Rhino-Laryngol. 270, 497-504. https://doi.org/10.1007/s00405-012-2018-3 (2013).

29. Rosengren, S. M., Colebatch, J. G., Young, A. S., Govender, S. \& Welgampola, M. S. Vestibular evoked myogenic potentials in practice: Methods, pitfalls and clinical applications. Clin. Neurophysiol. Pract. 4, 47-68. https://doi.org/10.1016/j.cnp.2019.01.005 (2019).

30. Chen, C.-W., Young, Y.-H. \& Tseng, H.-M. Preoperative versus postoperative role of vestibular-evoked myogenic potentials in cerebellopontine angle tumor. Laryngoscope 112, 267-271. https://doi.org/10.1097/00005537-200202000-00013 (2002).

31. Lachowska, M., Glinka, P. \& Niemczyk, K. Air-conducted and skull-tap cervical vestibular evoked myogenic potentials in determining nerve division involvement in vestibular schwannoma patients. Adv. Clin. Exp. Med. 27, 335-341. https://doi.org/10.17219/ acem/65484 (2018).

32. Manzari, L., Burgess, A. M., MacDougall, H. G. \& Curthoys, I. S. Vestibular function after vestibular neuritis. Int. J. Audiol. 52, 713-718. https://doi.org/10.3109/14992027.2013.809485 (2013).

33. Valame, D. A. \& Gore, G. B. Role of cervical vestibular evoked myogenic potentials (cVEMP) and auditory brainstem response (ABR) in the evaluation of vestibular schwannoma. Braz. J. Otorhinolaryngol. 83, 324-329. https://doi.org/10.1016/j.bjorl.2016. 04.003 (2017).

34. Tsutsumi, T., Tsunoda, A., Noguchi, Y. \& Komatsuzaki, A. Prediction of the nerves of origin of vestibular schwannomas with vestibular evoked myogenic potentials. Am. J. Otol. 21, 712-715 (2000).

35. Iwasaki, S. et al. Ocular vestibular evoked myogenic potentials to bone-conducted vibration in vestibular schwannomas. Otol. Neurotol. 31, 147-152. https://doi.org/10.1097/MAO.0b013e3181c0e670 (2010).

36. AAO-HNS. Committee on hearing and equilibrium guidelines for the evaluation of hearing preservation in acoustic neuroma (vestibular schwannoma). American Academy of Otolaryngology-Head and Neck Surgery Foundation, INC. Otolaryngol. Head Neck Surg. 113, 179-180. https://doi.org/10.1016/S0194-5998(95)70101-X (1995).

37. Rahne, T., Plontke, S. K., Vordermark, D., Strauss, C. \& Scheller, C. Hörklassen bei Patienten mit Vestibularisschwannom bei Verwendung deutschsprachiger Testverfahren. HNO https://doi.org/10.1007/s00106-020-00948-4 (2020).

38. Rahne, T., Weiser, C. \& Plontke, S. Neurofeedback-controlled comparison of the head elevation versus head rotation and headhand methods in eliciting cervical vestibular evoked myogenic potentials. Audiol. Neuro-otol. 19, 327-335. https://doi.org/10. $1159 / 000362661(2014)$.

39. Welgampola, M. S. \& Colebatch, J. G. Characteristics and clinical applications of vestibular-evoked myogenic potentials. Neurology 64, 1682-1688. https://doi.org/10.1212/01.WNL.0000161876.20552.AA (2005).

40. Koos, W. T., Spetzler, R. F. \& Böck, F. W. Microsurgery of cerebellopontine angle tumors. In Clinical Microneurosurgery (eds Koos, W. T. et al.) (Thieme Pub Sciences Group, 1976).

41. Matthews, B. W. Comparison of the predicted and observed secondary structure of T4 phage lysozyme. Biochim. Biophys. Acta 405, 442-451. https://doi.org/10.1016/0005-2795(75)90109-9 (1975).

42. Youden, W. J. Index for rating diagnostic tests. Cancer 3, 32-35. https://doi.org/10.1002/1097-0142(1950)3:1\%3c32::aid-cncr2 820030106\%3e3.0.co;2-3 (1950).

43. Caye-Thomasen, P., Dethloff, T., Hansen, S., Stangerup, S.-E. \& Thomsen, J. Hearing in patients with intracanalicular vestibular schwannomas. Audiol. Neuro-otol. 12, 1-12. https://doi.org/10.1159/000096152 (2007). 
44. West, N., Sass, H., Klokker, M. \& Cayé-Thomasen, P. Video head impulse test results in patients with a vestibular schwannomasensitivity and correlation with other vestibular system function tests, hearing acuity, and tumor size. Otol. Neurotol. https://doi. org/10.1097/MAO.0000000000002600 (2020).

45. Dilwali, S., Landegger, L. D., Soares, V. Y. R., Deschler, D. G. \& Stankovic, K. M. Secreted factors from human vestibular schwannomas can cause cochlear damage. Sci. Rep. 5, 18599. https://doi.org/10.1038/srep18599 (2015).

46. Soares, V. Y. R. et al. Extracellular vesicles derived from human vestibular schwannomas associated with poor hearing damage cochlear cells. Neuro Oncol. 18, 1498-1507. https://doi.org/10.1093/neuonc/now099 (2016).

47. McGarvie, L. A. et al. The video head impulse test (vHIT) of semicircular canal function-age-dependent normative values of VOR gain in healthy subjects. Front. Neurol. 6, 154. https://doi.org/10.3389/fneur.2015.00154 (2015).

48. Yang, C. J. et al. Quantitative analysis of gains and catch-up saccades of video-head-impulse testing by age in normal subjects. Clin. Otolaryngol. 41, 532-538. https://doi.org/10.1111/coa.12558 (2016).

49. Murofushi, T. \& Kaga, K. Vestibular Evoked Myogenic Potential (Springer, 2009).

50. Young, Y.-H., Huang, T.-W. \& Cheng, P.-W. Vestibular evoked myogenic potentials in delayed endolymphatic hydrops. Laryngoscope 112, 1623-1626. https://doi.org/10.1097/00005537-200209000-00018 (2002).

51. Manzari, L., MacDougall, H. G., Burgess, A. M. \& Curthoys, I. S. Selective otolith dysfunctions objectively verified. J. Vestib. Res. Equilib. Orient. 24, 365-373. https://doi.org/10.3233/VES-140537 (2014).

\section{Author contributions}

T.R.: conceptualization, methodology, software, formal analysis, writing_original draft, visualization, project administration. K.P.: validation, resources, writing-review and editing, supervision. L.F.: validation, writingreview and editing. C.S.: conceptualization, methodology, validation, investigation, resources, writing一review and editing, supervision.

\section{Funding}

Open Access funding enabled and organized by Projekt DEAL.

\section{Competing interests}

The authors declare no competing interests.

\section{Additional information}

Correspondence and requests for materials should be addressed to T.R.

Reprints and permissions information is available at www.nature.com/reprints.

Publisher's note Springer Nature remains neutral with regard to jurisdictional claims in published maps and institutional affiliations.

Open Access This article is licensed under a Creative Commons Attribution 4.0 International License, which permits use, sharing, adaptation, distribution and reproduction in any medium or format, as long as you give appropriate credit to the original author(s) and the source, provide a link to the Creative Commons licence, and indicate if changes were made. The images or other third party material in this article are included in the article's Creative Commons licence, unless indicated otherwise in a credit line to the material. If material is not included in the article's Creative Commons licence and your intended use is not permitted by statutory regulation or exceeds the permitted use, you will need to obtain permission directly from the copyright holder. To view a copy of this licence, visit http://creativecommons.org/licenses/by/4.0/.

(C) The Author(s) 2021 